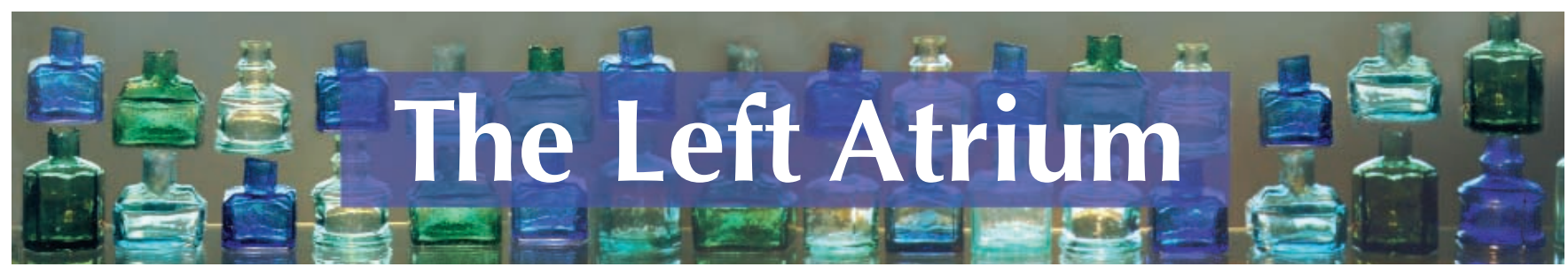

\section{A message in search of a method}

A different kind of care: the social pediatrics approach

Gilles Julien

Montréal: McGill-Queen's University Press; 2005

\$24.95 208 pp ISBN 0-7735-2801-6

$\mathrm{T}$ here's this spiky-haired kid wearing a camouflage T-shirt. He's got a ring on his ear and a sucker, which at first glance looks a lot like a cigarette, in his mouth. He's not looking right at you; rather, his eyes are diverted, but whether from embarrassment or lack of interest or distraction is hard to tell. Who is this kid?

"Who is this kid?" is the question that Dr. Gilles Julien, in his book $A$ Different Kind of Care: the Social Pediatrics Approach, challenges pediatricians to address. The boy I've just described appears on the book's cover, a poster child for the ambiguity of appearances. Is he from a poor family? How are his relationships with his parents? What does he think of himself? What does he need in order to be healthy and happy? Of course, none of these questions can be answered only by looking at a child, or even by conducting a traditional medical history and physical examination. But Julien would like you to believe that these are the most important questions to answer for many patients.

In this, he is preaching to the choir. I am still a trainee, but I have already had plenty of experience with children whose major problems are not medical per se but are issues of poverty, safety or emotional well-being. You don't have to go far in this business to meet kids whose serious medical problems are dwarfed by their lousy living situations. The idea that we need to understand these situations is no news at all. I suspect that anyone who chooses to read this book is very likely to be someone who has some experience in child health and understands the importance of social environment to children at least as well as
I do, and therefore has no need for a sermon on the issue.

But the need for understanding is only the point of departure for Julien, who practises pediatrics, teaches at McGill University and Université de Montréal, and has founded a number of community service groups for children. In this book, he would like to instruct the reader in obtaining an understanding of the needs of children. He makes this attempt in two parts, describing first the theory and then the practice of what he calls "social pediatrics."

As a guide, then, $A$ Different Kind of Care suffers from being poorly written and, consequently, difficult to read. This may, of course, be a function of its being a translation, and maybe it's a better read in the original French. (On the other hand, it's hard to imagine that "pediatricians should also develop deep tentaclelike roots in the child's milieu" is decent writing in any language.) A section on "The Theory of Social Pediatrics" is an intellectual ramble through some old ideas - namely holistic care and determinants of health - with some unnecessary terminology and meaningless diagrams thrown in for good measure. It reminded me of a bad undergraduate social sciences text. In addition, Julien is prone to defining terms as he pleases: "autonomy," for instance, is the "capacity to control your impulses and affects and become a sociable being" and "pervasive developmental disorder" has nothing to do with autism but is, rather, "great emotional suffer- ing." He also makes statements that lack both credibility and substantiation, such as "Many children who experience ... asthma ... are in search of an identity" and "[a three-year-old girl] seemed to have decided to stop living." The cumulative result of all this is an unconvincing book.

Leaving aside the merits of Julien or his translator as writers, $A$ Different Kind of Care has a much more substantial flaw, one that is perhaps fatal if Julien is hoping for his book to ignite substantial change in the way that pediatrics is practised. He begins by describing his four-step method for effective social pediatrics intervention: establishing a special relationship with a patient, exchanging information, decoding this information, and then acting accordingly. This all sounds very reasonable. Unfortunately, he doesn't tell us much about how to do any of it.

This is particularly true of the crucial "decoding" step. In the section entitled "The Practice of Social Pediatrics" Julien presents cases in which he has been involved, painting a portrait of a pediatrician with singular gifts of patience and insight. On this level, the book is an interesting foray into narrative medicine. But it's not much of a manual. Like many talented people, Julien assumes that things are as easy for others as they are for him. The text is full of words like "obviously," "clearly" and "realized"; it is short on the nitty-gritty of "figuring out" and "deducing." Beyond that, serendipity is evidently supposed to be one of a social pediatrician's clinical skills: thus the solution to a difficult case "appear[s]" in the form of "a ray of light." As more general advice, Julien tells us that "[e]verything comes at the right time, if we just know how to wait for it and have faith." I 
guess a social pediatrician had better not be a luckless agnostic.

What Julien wants us to do - fully understand the lives of troubled children - sounds like the subject of years of intensive training in, say, social work or child psychiatry. But he seems to frown on the idea of a pediatrician reaching out for such expert help:
They suggested sending him to a psychiatrist ... . Such an attitude ... has definite limitations and is liable to harm the child, since it immediately eliminates any attempt to seek explanatory causes and codes that can explain children's problems.

It's unlikely that any single book could concretely and usefully explain how to understand the inner lives of emotionally disturbed children. A Different Kind of Care certainly fails to do so - which is a shame, because we certainly need to be doing what Dr. Julien would like us to do.

\section{Paul Moorehead}

Pediatrics Resident

Memorial University of Newfoundland St. John's, Nfld.

\section{Disease as idea}

Lovers and livers: disease concepts in history

Jacalyn Duffin

Toronto: University of Toronto Press; 2005

299 pp \$27.50 ISBN 0-8020-3805-0

L overs and Livers is a compilation of lectures delivered in 2002 by Jacalyn Duffin, a hematologist and prolific medical historian based at Queen's University in Kingston, Ont. Through these lectures, she encourages us to look at diseases as "ideas influenced by the tastes and preoccupations of society." Duffin argues that it is "only when we already entertain cultural doubt about a trait or a behaviour" that we construe it as pathological and look to material causes to corroborate this view. To explore the "priority of culture over biology," Duffin proposes that vestiges of an old condition, lovesickness, may still affect the way we talk about illnesses such as erotomania, HIV and hepatitis C.

Understanding how illness, disease and culture are related to one another is the central problem in the emerging literature of "disease construction." The descriptive language of medicine is in constant flux, as it is recalibrated from generation to generation. Philosophical traditions cohabit this historical space in a beneficial exchange of ideas. For example, Descartes' Passions of the Soul can be read as a synthesis of Renaissance medical concepts with \& Cartesian theory. Over time, Cartesian notions of clear and distinct ideas and mind-body dualism have undergone a number of substantial revisions. Wittgenstein talks about "family resemblances" among words instead of clear and distinct ideas, and Eleanor
Rosch writes of conceptual prototypes. Mind-body dualism has been reformulated to encompass our contemporary view of consciousness. Similar transformations occurred in our concepts of truth, beauty and love. However, although philosophical and medical ideas have changed, disease construction must somehow anchor itself in an historical constant: the inevitability of human suffering.

Mirko Grmek, famed historian of medicine and Duffin's mentor, viewed illness concepts as groups of ideas in equilibrium at a given time, much like an assemblage of diverse organisms in a common habitat. As one way of thinking about illness falls into disuse (e.g., the concept of hysteria), another tends to take its place (e.g., multiple personality disorder). This notion has been thoroughly explored by Ian Hacking, using the pliable concepts of dissociation and multiple personality as modern reinventions of the soul. Syndromes such as these can be viewed as "containers" for many who don't fit the expected behavioural mould. Yet these containers are not pure abstractions. For each illness concept, there are real people experi- encing what Susan Sontag referred to as our "dual citizenship" in the realms of both sickness and health.

The medical link between hepatitis and sex has been discussed in the scientific literature. ${ }^{2}$ In her book, Duffin pairs the historical malady of lovesickness with a contemporary disease, infectious hepatitis.

She traces lovesickness back to ancient Egyptian and Greek love poems, in which the condition could prove fatal — as in the legend of Sappho plunging to her death over unrequited love. In medieval times, Persian doctors were likely the first to medicalize lovesickness, prescribing sex cures. Anecdotes from Renaissance medical writers and painters support the idea of love's ongoing power to cause illnesses with symptoms of melancholy, anorexia and even mania or suicide. After the Inquisition, sex cures may have fallen out of favour, and writers including Voltaire, Swift and Goethe linked lovesickness with venereal disease. Lovesickness was eventually divided into categories such as masturbation, homosexuality and pedophilia. Sexual perversity occupied Romantic fictional writers, while "[p]opular images of lovesick women ... [became] yet another tool of misogynist social control." Technological developments in surgery allowed procedures to cure a host of female complaints by ovariectomy, while doctors expanded their disease vocabulary to include homosexuality. In the 20th century, sex 